\title{
Comparative Effects of Cholestanol and Cholesterol on Hepatic Sterol and Bile Acid Metabolism in the Rat
}

\author{
S. Shefer, S. Hauser, G. Salen, F. G. Zaki, \\ J. Bullock, E. Salgado, and J. Shevitz \\ Department of Medicine, University of Medicine and Dentistry of \\ New Jersey, New Jersey Medical School, Newark, New Jersey \\ 07103; Veterans Administration Medical Center, East Orange, \\ New Jersey 07019; and Squibb Institute for Medical Research, \\ Princeton, New Jersey 08540
}

bstract. Large amounts of cholestanol, the $5 \alpha$-dihydro derivative of cholesterol are found in tissues of patients with the rare inherited sterol storage disease cerebrotendinous xanthomatosis. Although small amounts of cholestanol are present in virtually every tissue of normal man, little is known about its metabolism and effect on cholesterol and bile acid formation. The purpose of this study is to investigate the absorption and metabolism of cholestanol and its early effects on hepatic morphology and on the rate-limiting enzymes of cholesterol and bile acid biosynthesis. After 2 wk on a diet supplemented with $2 \%$ cholestanol, total liver sterol content increased by $48 \%$ (3.26 vs. $2.20 \mathrm{mg} / \mathrm{g}$ ), and resulted in a significant rise in hepatic cholestanol concentration to $1.4 \mathrm{mg} / \mathrm{g}$. However, cholestanol was less efficiently absorbed from the intestine than cholesterol and interfered with cholesterol absorption. Furthermore, hepatic hydroxymethylglutaryl-coenzyme A (HMG-CoA) reductase activity rose 2.6-fold (from 150.3 to $397.0 \mathrm{pmol} / \mathrm{mg}$ per min) during cholestanol feeding, and was associated with a marked proliferation of the smooth endoplasmic reticulum of the centrilobular areas. In addition, significant amounts of allocholic acid (16\%) and allochenodeoxycholic acid (5\%) were formed from cholestanol and excreted in the bile. These results show that cholestanol is absorbed from the intestine, interferes with cholesterol absorption, and is deposited in the liver. However, in contrast to cholesterol, cholestanol feeding was associated with a marked elevation of HMG-CoA

Address correspondence to Dr. Shefer, New Jersey Medical School. Received for publication 3 May 1984.

J. Clin. Invest.

(C) The American Society for Clinical Investigation, Inc. 0021-9738/84/11/1773/09 \$1.00

Volume 74, November 1984, 1773-1781 reductase activity. Thus, despite structural similarity between cholesterol and its $5 \alpha$-saturated derivative, cholestanol does not exert feedback inhibition on hepatic cholesterol biosynthesis.

\section{Introduction}

Small amounts of the saturated sterol, $5 \alpha$-cholestan- $3 \beta$-ol (cholestanol, Fig. 1) have been detected in all sterol fractions of mammalian origin (1). Cholestanol is structurally similar to cholesterol and like cholesterol appears both in the free and esterified forms. Relatively low concentrations of cholestanol $(0.2-0.6 \%)$ were detected in the sterol fraction of human gallstones and aortas; somewhat larger amounts (0.4-1.0\%) were present in the serum of man, rabbit and dog; and relatively high concentration $(2-10 \%)$ occurred in intestinal wall, liver, and adrenals of rabbits and guinea pigs (2). Recently, large amounts of cholestanol have been detected in tissues and plasma of patients with the rare inherited sterol storage diseases, cerebrotendinous xanthomatosis and sitosterolemia with xanthomatosis (3). Despite its widespread distribution, little is known about cholestanol metabolism, its effect on cholesterol synthesis, and potential for bile acid formation. However, it has been shown that cholestanol can be transformed into allobile acids, the $5 \alpha$-saturated bile acids by a pathway similar to that of the normally occurring primary $5 \beta$-bile acids $(4,5)$.

The present study was designed to examine the absorption, distribution, and metabolic effects of cholestanol on the hepatic rate-controlling enzymes of cholesterol and bile acid synthesis. Alterations in the hepatic ultrastructure related to the administration of cholestanol and cholesterol were also investigated.

\section{Animals and diets}

Sprague-Dawley male rats of initial body weight of 130-150 g were kept individually in metabolism cages to prevent coprophagy. They were fed a stock diet consisting of ground Purina rat chow (5001) supplemented with 5\% corn oil. This diet contained $0.014 \%$ cholesterol, $0.034 \%$ plant sterols, and only trace amounts of cholestanol. When required, sterols 
<smiles>CC(C)CCCC1CCC2C1CCC1C2CCC2CC(O)CCC21C</smiles>

Figure 1. Structure of $5 \alpha$-cholestan$3 \beta$-ol (cholestanol).

were added to this diet at a level of $2 \%$. The sterols were incorporated into the diets from an ether solution as described previously (6). The rats were fed the diets and drinking water ad lib. for $14 \mathrm{~d}$. Weight and food intake were recorded daily, and any animal that differed by $>10 \%$ from the average of the control group was excluded from the study.

\section{Experimental design}

Three groups of rats (five animals in each group) were fed (a) control diet, $(b)$ diet supplemented with $2 \%$ cholestanol, and (c) diet supplemented with $2 \%$ cholesterol. At the end of the experimental period the animals were anesthetized with sodium pentobarbital (Fort Dodge Laboratories, Inc., Fort Dodge, IA). The common bile duct was cannulated and bile was collected for a period of 30-60 min. In addition, blood samples were taken from each animal. The rats were then killed by exsanguination and the livers were excised, weighed, and prepared for sterol, enzymatic, and histologic evaluation. Measurements were carried out to determine HMG-CoA reductase and cholesterol $7 \alpha$-hydroxylase activity, biliary, fecal, serum, and hepatic sterol concentrations, and biliary bile acid composition.

In addition, two groups of rats were treated with cholesterol or cholestanol as described above, with the exception that after 2 wk the rats were given an oral tracer dose of $\left[1,2-{ }^{3} \mathrm{H}\right]$ cholesterol and $\beta-\left[4^{14}-\mathrm{C}\right]$ sitosterol for the measurement of intestinal cholesterol absorption. These results were compared with those of a control group. Another control group was given an oral tracer dose of $\left[1,2-{ }^{3} \mathrm{H}\right]$ cholestanol and $\beta-\left[4^{14}-C\right]$ sitosterol for the measurement of intestinal cholestanol absorption.

\section{Experimental procedures}

Materials. Cholesterol United States Pharmacopeia (U.S.P.) was recrystallized from ethanol and used without further purification (contained $<0.1 \%$ cholestanol). Cholestanol was purchased from Sigma Chemical Co., St. Louis, MO and was 99\% pure. The purity of both sterols was established by gasliquid chromatography (GLC).

Labeled compounds. $\left[4-{ }^{14} \mathrm{C}\right]$ Cholesterol $58 \mathrm{mCi} / \mathrm{mmol}(\mathrm{New}$ England Nuclear, Boston, MA) was diluted with unlabeled cholesterol (Sigma Chemical Co.) to the required specific radioactivity and purified by chromatography on a silicic acid column (Bio-Sil, Bio-Rad Laboratories, Richmond, CA) by elution with increasing amounts of ethyl ether in hexane (7). The purified cholesterol contained $<0.06 \% 7 \alpha$-hydroxycholesterol, and was used as a substrate for the cholesterol $7 \alpha$-hydroxylase assay. $\left[1,2-{ }^{3} \mathrm{H}\right] \mathrm{Cholesterol} \mathrm{was} \mathrm{purchased} \mathrm{from}$ New England Nuclear and was purified by chromatography on a column of silicic acid impregnated with silver nitrate (8). The purified product contained $<0.04 \%$ cholestanol, and was used in the absorption studies. $\beta-\left[4-{ }^{14} \mathrm{C}\right]$ sitosterol was purchased from Amersham Corp., Arlington Heights, IL, and purified by thin-layer chromatography (TLC) ${ }^{1}$ before use (9). R,S-[3$\left.{ }^{14} \mathrm{C}\right] \mathrm{HMG}-\mathrm{CoA}$ was prepared by reacting $\left[3-{ }^{14} \mathrm{C}\right] \mathrm{HMG}$ anhydride with coenzyme A SH (10). $\left[1,2-{ }^{3} \mathrm{H}\right]$ Cholestanol was prepared from $\left[1,2-{ }^{3} \mathrm{H}\right]$ cholesterol (New England Nuclear) by catalytic hydrogenation using $\mathrm{PtO}_{2}$ on charcoal as catalyst. The cholestanol was purified by argentation TLC and it contained $<0.3 \%$ cholesterol (11).

Reference compounds. Cholic, deoxycholic, chenodeoxycholic, and lithocholic acids were purchased from CalbiochemBehring Corp., San Diego, CA. $\alpha$ - and $\beta$-muricholic acids were synthesized according to Hsia et al. $(12,13)$. Allocholic and allochenoxydeocholic acids were a gift from Dr. W. H. Elliott, St. Louis, MO. $7 \alpha$ - and $7 \beta$-hydroxycholesterol were prepared by reduction of 7-ketocholesterol (Schwarz/Mann, Orangeburg, NY) with sodium borohydride (7).

$G L C$. Cholesterol and cholestanol were analyzed and quantitated by GLC on $180 \times 0.4-\mathrm{cm}$ columns packed with SP-1000 (Applied Science Laboratories, Inc., State College, PA). Column temperature was $220^{\circ} \mathrm{C}$, and $\mathrm{N}_{2}$ flow $30 \mathrm{ml} / \mathrm{min}$ (model 5840A gas chromatograph, Hewlett Packard Co., Palo Alto, CA). The retention times, relative to $5 \alpha$-cholestane (7.31 min), were cholesterol, 7.11 and cholestanol, 6.19. Bile acids were determined as the trimethylsilyl-ether methyl derivatives on Hi-EFF 8BP columns (Applied Science Laboratories), column temperature was $240^{\circ} \mathrm{C}$, and $\mathrm{N}_{2}$ flow $35 \mathrm{ml} / \mathrm{min}$. The retention times, relative to $5 \alpha$-cholestane $(3.2 \mathrm{~min})$, were: cholic acid, 1.53; chendeoxycholic acid, 2.54; allocholic acid, 1.27; and allochendeoxycholic acid, 2.07.

Mass spectra. Mass spectra of the bile acids were obtained with a Varian MAT-III gas chromatograph-mass spectrometer (Varian Associates, Palo Alto, CA) at an ion source pressure of $2-3 \times 10^{-6} \mathrm{~mm}$ and an electron energy of $70 \mathrm{eV}$, as described previously (14).

\section{Methods}

Determination of biliary sterols and bile acids. Biliary sterols and bile acids were isolated, identified, and quantitated by a combination of TLC and GLC-mass spectroscopy, as described previously $(15,16)$.

Fecal sterol and bile acid analysis. Feces and intestinal contents

1. Abbreviations used in this paper: GLC, gas-liquid chromatography; HMG CoA, hydroxymethylglutaryl-coenzyme A; TLC, thin-layer chromatography. The following systematic names are given to compounds referred to by trivial names: cholesterol, 5 -cholesten-3 $\beta$-ol; cholestanol, $5 \alpha$-cholestan- $3 \beta$-ol; coprostanol, $5 \beta$-cholestan- $3 \beta$-ol; $7 \alpha$-hydroxycholesterol, 5-cholesten-3 $\beta, 7 \alpha$-diol; $\beta$-sitosterol, $24 \beta$-ethyl-5-cholesten-3 $\beta$ ol; cholic acid, $3 \alpha, 7 \alpha, 12 \alpha$-trihydroxy-5 $\beta$-cholanoic acid; $\beta$-muricholic acid, $3 \alpha, 6 \beta, 7 \beta$-trihydroxy-5$\beta$-cholanoic acid; allocholic acid, $3 \alpha, 7 \alpha, 12 \alpha-$ trihydroxy-5 $\alpha$-cholanoic acid; allochenodeoxycholic acid, $3 \alpha, 7 \alpha$-dihydroxy-5 $\alpha$-cholanoic acid. 
were pooled, dried, and extracted with ethanol in a Soxhlet extractor for $48 \mathrm{~h}$. After separation of the acidic steroids from the neutral ones, saponification under pressure, extraction, and methylation (17) bile acid composition was determined by GLC.

Serum sterol analysis. 1 vol of serum $(0.1-0.5 \mathrm{ml})$ was diluted with $9 \mathrm{vol}$ of $0.1 \mathrm{M} \mathrm{NaOH}$ in $0.9 \% \mathrm{NaCl}$ and percolated through a column of Amberlite XAD-2 (Servachrom XAD-2, 100-200 $\mu \mathrm{m}$, Serva, Heidelberg, Federal Republic of Germany) (18). The eluate was evaporated and sterol concentrations were determined by GLC.

Cholesterol determination in hepatic microsomes. Known aliquots of microsomal suspensions were refluxed for $3 \mathrm{~h}$ with $25 \%$ ethanolic $\mathrm{KOH}$ (19). Cholesterol and cholestanol were extracted with $n$-hexane and analyzed by GLC using $5 \alpha$-cholestane as internal standard as described in "Experimental Procedures".

Determination of free and esterified sterols in the liver. The lipids in $1 \mathrm{ml}$ liver homogenate were extracted in $20 \mathrm{ml}$ of chloroform/ methanol, 2:1 (11). The solvent was evaporated and the lipid residue was divided into two portions. Free sterols were determined by GLC, while total sterol content was determined in the same manner after alkaline hydrolysis (19). The percent of esterified sterols was calculated from the difference of these two values.

Histology. For light microscopic examination, buffered formalinfixed and paraffin-embedded $6-\mu \mathrm{m}$-thick sections of liver specimens were stained with hematoxylin and eosin and with other special stains such as Masson's trichrome for connective tissue. For electron microscopic studies, small pieces of the liver were fixed in ice-cold s-collidine-buffered $1 \%$ osmium tetroxide. After dehydration through a series of graded concentrations of alcohol, they were embedded in Epon $812(20)$. Sections $0.5-\mu \mathrm{m}$ thick and stained with toluidine blue were examined to depict areas from the periportal and centrilobular areas of the hepatic lobules. Ultra thin sections from such areas were stained with uranyl acetate and lead citrate (21) and examined in Zeiss 9S electron microscope.

Measurement of intestinal absorption. The intestinal absorption of cholesterol was measured in rats treated with cholestanol or cholesterol (five rats per group) and compared to those of the control group. At the end of the experimental period, a tracer dose of $\left[1,2-{ }^{3} \mathrm{H}\right]$ cholesterol and $\beta$-[14- $\left.{ }^{14} \mathrm{C}\right]$ sitosterol $\left({ }^{3} \mathrm{H} /{ }^{14} \mathrm{C}, 4: 1\right)$ was administered orally to the rats. The ratio of ${ }^{3} \mathrm{H} /{ }^{14} \mathrm{C}$ was determined in the fecal samples collected and compared with that of the dietary dose. The percent absorption was calculated according to Borgström et al. (22). Cholestanol absorption was determined in the control group in a similar manner, with the exception that $\left[1,2-{ }^{3} \mathrm{H}\right]$ cholestanol was used in place of the tritiated cholesterol.

Statistical analysis. Data are presented as mean \pm SD unless otherwise indicated. The significance was evaluated by $t$ test.

Enzyme assays. The remainder of the liver tissue (kept at $0^{\circ}-5^{\circ} \mathrm{C}$ ) was used immediately for preparation of the microsomal fraction. In order to eliminate as far as possible variation due to the diurnal rhythm of enzyme activity, all animals were killed by 9 to 11 a.m., i.e., during the diurnal minimum $(23,24)$. The specific activity of hepatic microsomal hydroxymethylglutaryl-coenzyme A (HMG-CoA) reductase was determined in the presence of $\mathrm{NaF}$ exactly as described by Rodwell et al. (25). The specific activity of cholesterol $7 \alpha$-hydroxylase was determined in acetone powder preparations obtained from the microsomal fractions and contained virtually no endogenous cholesterol (19).

\section{Results}

Hepatic sterol content. Table I shows the effect of cholestanol and cholesterol feeding on hepatic sterol concentration. When a diet that contained $2 \%$ cholestanol was fed there was a $48 \%$ increase in total sterol concentration $(3.26 \pm 0.2$ vs. $2.2 \pm 0.17$ $\mathrm{mg} / \mathrm{g}$ liver; $P<0.00001)$. The rise in hepatic sterols was entirely accounted for by the deposition of absorbed dietary cholestanol and was associated with a small decline in hepatic cholesterol content. The cholestanol content increased 35-fold from $0.04 \pm 0.003$ to $1.40 \pm 0.09 \mathrm{mg} / \mathrm{g}$ liver. When a diet that contained $2 \%$ cholesterol was fed, total hepatic sterol concentration increased fourfold over control $(8.66 \pm 0.4 \mathrm{vs}$. $2.20 \pm 0.17$ $\mathrm{mg} / \mathrm{g}$ liver), and $\sim 2.5$ times over cholestanol feeding. The amount of cholestanol per gram of liver did not differ from control.

In this table are also listed the proportions of free and esterified sterols following each dietary regimen. In the control animals, $70 \%$ of the cholesterol and $75 \%$ of the cholestanol was in the free form. During cholestanol feeding the percentage of esterified cholesterol and cholestanol increased by $5-6 \%$ despite the large increase in cholestanol concentration. As expected, during cholesterol feeding $84 \%$ of the cholesterol was

Table I. Content and Distribution of Esterified and Free Sterols in the Liver*

\begin{tabular}{|c|c|c|c|c|c|c|}
\hline \multirow[t]{2}{*}{$\begin{array}{l}\text { Additions to diet } \\
(2 \mathrm{wk})\end{array}$} & \multicolumn{3}{|c|}{ Hepatic cholestanol } & \multicolumn{3}{|c|}{ Hepatic cholesterol } \\
\hline & $m g / g$ liver & & $\%$ & $\mathrm{mg} / \mathrm{g}$ liver & & $\%$ \\
\hline None & $0.04 \pm 0.003$ & $\begin{array}{l}\text { Free } \\
\text { Esterified }\end{array}$ & $\begin{array}{l}74.9 \pm 5.6 \\
25.1 \pm 2.1\end{array}$ & $2.16 \pm 0.12$ & $\begin{array}{l}\text { Free } \\
\text { Esterified }\end{array}$ & $\begin{array}{l}70.3 \pm 3.2 \\
29.1 \pm 1.5\end{array}$ \\
\hline 2\% Cholestanol & $1.40 \pm 0.09$ & $\begin{array}{l}\text { Free } \\
\text { Esterified }\end{array}$ & $\begin{array}{l}68.7 \pm 4.1 \\
31.3 \pm 2.3\end{array}$ & $1.86 \pm 0.13$ & $\begin{array}{l}\text { Free } \\
\text { Esterified }\end{array}$ & $\begin{array}{l}65.2 \pm 3.4 \\
34.8 \pm 1.5\end{array}$ \\
\hline 2\% Cholesterol & $0.11 \pm 0.006$ & $\begin{array}{l}\text { Free } \\
\text { Esterified }\end{array}$ & $\begin{array}{l}69.4 \pm 5.0 \\
30.6 \pm 2.6\end{array}$ & $8.55 \pm 0.43$ & $\begin{array}{l}\text { Free } \\
\text { Esterified }\end{array}$ & $\begin{array}{l}16.1 \pm 1.2 \\
83.9 \pm 3.9\end{array}$ \\
\hline
\end{tabular}

* The data in the table represent the average of five rats for each group. (mean \pm SD). $¥$ Ground Purina chow supplemented with $5 \%$ corn oil. 
accumulated in the liver in the esterified form while only $31 \%$ of the cholestanol was esterified. These results show that in untreated animals most of the sterols in the liver are present as free sterols and only $30 \%$ of the total cholesterol and $25 \%$ of the cholestanol are in the ester form. During sterol feeding, hepatic esterification is activated, and exhibits greater affinity towards cholesterol.

Intestinal absorption. Measurements of cholesterol and cholestanol absorption were made by the isotope ratio technique of Borgström et al. (22), utilizing $\beta-\left[4-{ }^{14} \mathrm{C}\right]$ sitosterol as the unabsorbed internal standard and either $\left[1,2-{ }^{3} \mathrm{H}\right]$ cholesterol or $\left[1,2-{ }^{3} \mathrm{H}\right]$ cholestanol as the test sterols. Cholestanol absorption was compared to that of cholesterol in the control rats. Table II illustrates that cholestanol was less efficiently absorbed than cholesterol, 14.0 vs. $44.8 \%$, respectively. Thus, the intestine discriminates against the structurally similar cholestanol. When cholesterol absorption of the control rats was compared to that of rats fed a diet supplemented with $2 \%$ cholestanol, there was a $20 \%$ decrease in cholesterol absorption which was statistically significant, and suggests that the dietary cholestanol competes and interferes with cholesterol absorption. However, when the diet contained $2 \%$ cholesterol, the proportion of cholesterol absorbed was similar to that observed in the control rats.

Serum and biliary sterol concentration. The measurements of serum cholesterol and cholestanol concentrations are listed in Table III. After cholesterol or cholestanol feeding, there was no significant difference in total sterol concentration. However, unlike serum cholesterol levels that remained unchanged with cholesterol feeding, there was a sixfold rise in serum cholestanol concentration when this saturated sterol was fed. But despite

Table II. Intestinal Sterol Absorption*

\begin{tabular}{|c|c|c|c|c|}
\hline \multirow[b]{2}{*}{$\begin{array}{l}\text { Additions to } \\
\text { diet } ¥ \text { (2 wk) }\end{array}$} & \multicolumn{2}{|l|}{ Cholesterol } & \multicolumn{2}{|l|}{ Cholestanol } \\
\hline & $\begin{array}{l}\text { Fecal } \\
\text { excretion§ } \\
\left({ }^{14} \mathrm{C} /{ }^{3} \mathrm{H}\right)\end{array}$ & Absorption" & $\begin{array}{l}\text { Fecal } \\
\text { excretionf } \\
\left({ }^{14} \mathrm{C} /{ }^{3} \mathrm{H}\right)\end{array}$ & Absorption" \\
\hline & & $\%$ & & $\%$ \\
\hline None & 1.73 & $44.8 \pm 2.5$ & 0.54 & $14.0 \pm 2.0$ \\
\hline 2\% Cholestanol & 1.39 & $36.0 \pm 1.6^{* *}$ & & \\
\hline $2 \%$ Cholesterol & 1.64 & $42.5 \pm 2.3 \ddagger \ddagger$ & & \\
\hline
\end{tabular}

* The data in the table represent the average of five rats for each group (mean $\pm \mathrm{SD}$ ).

$\ddagger$ Ground Purina chow supplemented with $5 \%$ corn oil.

$\S\left[1,2-{ }^{3} \mathrm{H}\right]$ cholesterol $(10 \mu \mathrm{mol}),\left[4-{ }^{14} \mathrm{C}\right] \beta$-sitosterol $(10 \mu \mathrm{mol})$ and $200 \mu \mathrm{mol}$ of

triolein were administered orally to the experimental rats, and the ratio ${ }^{14} \mathrm{C} /{ }^{3} \mathrm{H}$ of the percent radioactivity recovered in the feces was calculated.

"Intestinal cholesterol and cholestanol absorption was determined according to Borgström et al. (24).

I $\left[1,2-{ }^{3} \mathrm{H}\right]$ cholestanol $(10 \mu \mathrm{mol})$ was substituted for $\left[1,2-{ }^{3} \mathrm{H}\right]$ cholesterol when intestinal cholestanol absorption was determined.

** Differs significantly from control values $(P<0.0001)$.

¥ Does not differ significantly from control values.
Table III. Effect of Diet on Biliary and Serum Concentrations*

\begin{tabular}{|c|c|c|c|c|}
\hline \multirow{3}{*}{$\begin{array}{l}\text { Additions to } \\
\text { diet } \text { ( } 2 \mathrm{wk} \text { ) }\end{array}$} & \multicolumn{4}{|c|}{ Sterol concentration } \\
\hline & \multicolumn{2}{|l|}{ Serum } & \multicolumn{2}{|l|}{ Bile } \\
\hline & Total & Cholestanol & Total & Cholestanol \\
\hline & $m g / d l$ & $\%$ & $m g / m l$ & $\%$ \\
\hline None & $80 \pm 3.8$ & $0.12 \pm 0.007$ & $0.145 \pm 0.009$ & $0.13 \pm 0.008$ \\
\hline $2 \%$ Cholestanol & $78 \pm 4.7$ & $0.70 \pm 0.04 \S$ & $0.150 \pm 0.01$ & $0.47 \pm 0.02 \S$ \\
\hline $2 \%$ Cholesterol & $85 \pm 4.0$ & $0.11 \pm 0.005$ & $0.139 \pm 0.008$ & $0.15 \pm 0.009$ \\
\hline
\end{tabular}

* Biliary and serum sterol concentrations were determined as described in "Experimental Procedures". The data represent the average of five rats for each group (mean $\pm \mathrm{SD}$ ).

¥ Ground Purina chow supplemented with $5 \%$ corn oil.

$\S$ Differs significantly from control values $(P<0.00005)$.

the large intake of cholestanol, the increment amounted to only $0.58 \mathrm{mg} / \mathrm{dl}$.

Biliary sterol concentration (Table III) did not increase despite the marked rise in hepatic sterol levels during the experimental feeding period (Table I). However, there was an almost fourfold increase in the percent of cholestanol in the biliary sterols after its feeding.

Biliary bile acid composition. The effect of either cholestanol or cholesterol feeding on biliary bile acid composition is shown in Fig. 2. In the control rats, cholic acid constituted $77 \%$ of the biliary bile acids, chenodeoxycholic acid $14 \%$, the muricholic acids $9 \%$, and there were no detectable allo-bile acids. After cholesterol feeding there was no significant difference in biliary bile acid composition. However, when cholestanol was fed there was an increased formation and excretion of allocholic acid $(16 \%)$ and allochenodeoxycholic acid $(5 \%)$, the respective $5 \alpha$-saturated bile acids. These allo-bile acids are produced

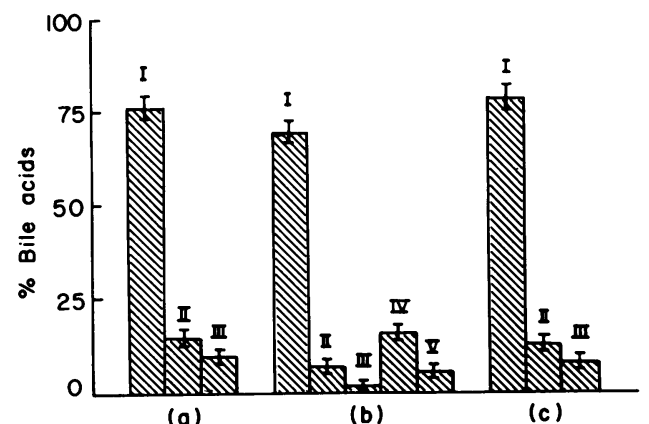

Figure 2. The effect of diet on biliary bile acid composition. The rats received either regular rat Purina chow, control (a), or were supplemented with $2 \%$ cholestanol (b), or $2 \%$ cholesterol (c). The bars represent percentage of (I) cholic acid, (II) chenodeoxycholic acid, (III) $\alpha$ - and $\beta$-muricholic acids, (IV) allocholic acid, and (V) allochenodeoxycholic acid. 
from cholestanol (26), and their increased formation probably reflects the large hepatic pool of cholestanol.

The rate-controlling enzymes of cholesterol and bile acid biosynthesis. In Table IV are shown the specific activities of hepatic HMG-CoA reductase. As expected, during cholesterol feeding there was a fivefold drop in the activity of HMG-CoA reductase as compared with the control group. In contrast, during cholestanol feeding, HMG-CoA reductase activity increased 2.6-fold as compared with the control values, and was 13 times higher than HMG-CoA reductase activity during cholesterol feeding. These results suggest that the absorbed cholestanol might stimulate hepatic HMG-CoA reductase activity, and unlike cholesterol, does not exert feedback inhibition on this rate controlling enzyme.

It is noteworthy that microsomal cholesterol levels fell by $\sim 8 \%$ when cholestanol was fed, and although there was a small increase in microsomal cholestanol content, total sterol values did not differ significantly from those of the control values. In contrast, cholesterol feeding led to a substantial rise in microsomal cholesterol content. Thus, the inhibition of hepatic HMG-CoA reductase activity may be modulated through the concentration of microsomal cholesterol.

As to cholesterol $7 \alpha$-hydroxylase (Table V), neither cholestanol nor cholesterol feeding affected the activity of this key rate controlling enzyme. It is important to note that these measurements of cholesterol $7 \alpha$-hydroxylase activity were made with acetone treated microsomal preparations in which the endogenous sterols are completely removed (19). The sterol content of the acetone powder prepared from hepatic microsomes of rats treated with cholestanol or cholesterol was similar to that of rats fed the control diet, and was as low as $0.38-$ $0.43 \mathrm{nmol} / \mathrm{mg}$ of protein, although the sterol content of the different microsomal preparations varied (Table IV).

Fecal sterols. During cholestanol feeding, this $5 \alpha$-dihydro derivative of cholesterol was the major stanol excreted in the feces (Table VI), which is consistent with its reduced intestinal

Table IV. Hepatic HMG-CoA Reductase Activity*

\begin{tabular}{llll}
\hline $\begin{array}{l}\text { Additions to } \\
\text { control diet } \\
(2 \mathrm{wk})\end{array}$ & $\begin{array}{l}\text { HMG-CoA } \\
\text { reductase } \\
\text { activity }\end{array}$ & \multicolumn{2}{l}{ Microsomal sterol concentration } \\
\cline { 4 - 5 } & $\begin{array}{l}\text { pmol/mg } \\
\text { protein } / \mathrm{min}\end{array}$ & Cholestanol & Cholesterol \\
\hline & $150.3 \pm 4.5$ & Trace $\$$ & \\
None & $397.0 \pm 15.2$ & $0.022 \pm 0.0007$ & $84.7 \pm 3.2$ \\
$2 \%$ Cholestanol & $30.1 \pm 1.3$ & Trace & $78.2 \pm 3.0^{\prime \prime}$ \\
$2 \%$ Cholesterol & & & $118.1 \pm 3.6 \pi$
\end{tabular}

* Hepatic HMG-CoA reductase activity was determined as described in "Experimental Procedures". The data in the table represent the average of five rats for each group (mean $\pm \mathrm{SD}$ ).

¥ Ground Purina chow supplemented with $5 \%$ corn oil.

$\S<0.001 \mathrm{nmol} / \mathrm{mg}$ protein.

"Does not differ significantly from control values $(P>0.5)$

I Differs significantly from control values $(P<0.0005)$.
Table V. Hepatic Cholesterol $7 \alpha-$ Hydroxylase Activity*

\begin{tabular}{|c|c|c|c|}
\hline \multirow{2}{*}{$\begin{array}{l}\text { Additions to } \\
\text { control diet } \neq \\
(2 \mathrm{wk})\end{array}$} & \multirow{2}{*}{$\begin{array}{l}\text { Cholesterol } \\
7 \alpha \text {-hydroxylase } \\
\text { activity }\end{array}$} & \multicolumn{2}{|c|}{$\begin{array}{l}\text { Sterol concentration in } \\
\text { acetone powder }\end{array}$} \\
\hline & & Cholestanol & Cholesterol \\
\hline & $\begin{array}{l}\mathrm{pmol} / \mathrm{mg} \\
\text { protein/min }\end{array}$ & \multicolumn{2}{|c|}{ nmol/mg protein } \\
\hline None & $11.7 \pm 1.2$ & $<0.01$ & $0.41 \pm 0.02$ \\
\hline $2 \%$ Cholestanol & $11.0 \pm 1.1 \S$ & $<0.01$ & $0.38 \pm 0.03 \S$ \\
\hline $2 \%$ Cholesterol & $12.3 \pm 1.3 \S$ & $<0.01$ & $0.43 \pm 0.03 \S$ \\
\hline
\end{tabular}

* The data in the table represent the average of five rats for each group (mean $\pm \mathrm{SD}$ ). Cholesterol $7 \alpha$-hydroxylase activity was determined in microsomal preparations in the absence of endogenous cholesterol as described in "Experimental Procedures".

‡ Ground Purina chow supplemented with $5 \%$ corn oil.

$\S$ Does not differ significantly from control values.

absorption (Table II). Of interest is the increased excretion of cholesterol and its bacterial metabolite, coprostanol, which was 2.5 times greater than that excreted in the control rats, 22.8 vs. $9.1 \mathrm{mg} / \mathrm{d}$, respectively. This observation indicates that cholestanol interfered with cholesterol absorption and is consistent with the results in Table II. During cholesterol feeding, this sterol was the predominant one in the feces, but the quantity of the excreted cholesterol and coprostanol (165.4 $\mathrm{mg} / \mathrm{d})$ was smaller than that of cholestanol $(230.1 \mathrm{mg} / \mathrm{d})$ when it was fed. This finding is additional evidence that cholesterol was more efficiently absorbed than cholestanol. Furthermore, more bile acids were excreted following cholesterol feeding than after cholestanol or control periods.

Histologic findings. Light microscopic examination of liver sections taken from rats given either cholestanol or cholesterol appeared quite normal. However, electron microscopic examination of the same livers depicted some but nondeleterious alterations in the hepatocytic organelles.

In cholestanol-fed rats, hepatocytes of the periportal areas (zone 1 of Rappaport) appeared normal, with mild increase in

Table VI. Fecal Sterol and Bile Acid Outputs*

\begin{tabular}{lllll}
\hline & \multicolumn{2}{l}{ Fecal sterols } & & \\
\cline { 2 - 3 } $\begin{array}{l}\text { Additions to } \\
\text { diet¥ }(2 \mathrm{wk})\end{array}$ & Cholestanol & Cholesterol & Coprostanol & $\begin{array}{l}\text { Total bile } \\
\text { acids }\end{array}$ \\
\hline & & $m g / 24 h$ & & $m g / 24 h$ \\
& & $5.17 \pm 0.24$ & $3.94 \pm 0.24$ & $17.44 \pm 0.86$ \\
None & $<0.1$ & $17.90 \pm 0.54$ & $4.93 \pm 0.26$ & $16.71 \pm 0.51$ \\
$2 \%$ Cholestanol & $230.1 \pm 11.5$ & $132.1 \pm 6.1$ & $33.33 \pm 1.6$ & $49.96 \pm 2.6$
\end{tabular}

* Fecal sterol and bile acid outputs were determined as described in "Experimental Procedures". The data in this table represent an average of five rats for each group (mean $\pm S D$ ).

‡ Ground Purina chow supplemented with $5 \%$ corn oil. 
the smooth endoplasmic reticulum (Fig. 3). In contrast, a marked increase of the vesicles of this organelle (Fig. 4) was observed in hepatocytes of the central areas of the hepatic lobule (zone III of Rappaport), which indicates higher activity of the biotransformation enzyme systems.

On the other hand, the livers of rats fed cholesterol revealed less response of the smooth endoplasmic reticulum, which appeared normal in hepatocytes of the periportal areas (Fig. 5 ) and more vesicular, and moderately increased in the centrilobular hepatocytes (Fig. 6). The Golgi vesicles often appeared dilated, and mild fatty infiltration was frequently noted (Fig. 5). Some of the centrilobular hepatocytes revealed a significant increase in the peroxisomes, a phenomenon often seen in increased bile acid metabolism (Fig. 6).

\section{Discussion}

The results of this investigation show a number of differences in the metabolism of cholesterol and cholestanol in the rat. Both are absorbed, as evidenced by the rise in hepatic sterol concentration (Table I), and by the measurements of intestinal absorption (Table II). However, cholesterol was more readily absorbed than cholestanol, 44.8 vs. $14.0 \%$, respectively. This was reflected by the nearly fourfold increase in hepatic sterol content after cholesterol administration, whereas the administration of the same amount of cholestanol resulted in only $48 \%$ increase (Table I). Furthermore, the $24 \mathrm{~h}$-fecal excretion data (Table VI) reflect the same differential absorption. A significantly smaller fraction of the dietary sterol was excreted in the feces following cholesterol feeding $(165.4 \mathrm{mg} / \mathrm{d})$ than that after cholestanol feeding $(230.1 \mathrm{mg} / \mathrm{d})$. Thus, the intestinal transport system appears to recognize the structural difference between the two compounds. Moreover, the 2.5 -fold increase in the excretion of endogenous cholesterol above control $(22.8$ vs. $9.1 \mathrm{mg} / \mathrm{d}$, respectively) brought about by exogenous cholestanol suggests that the dietary stanol competes favorably for the active absorption mechanism in the intestine.

Current theory states that the rate limiting step for sterol transport is esterification with long chain fatty acid residues. Perhaps cholestanol is a less suitable substrate for intestinal acyl CoA:cholesterol acyltransferase, and as a consequence would slow the entire process of esterification and thereby transport of cholesterol by the intestine. It might also be pointed out that hepatic esterification was much less efficient for cholestanol in contrast to cholesterol (Table I; 31.3 vs. $83.9 \%$, respectively), despite the enhanced sterol absorption noted during both feeding regimens.

It is noteworthy that total sterol levels in bile and serum remained unchanged (Table III). However, the relative proportion of cholestanol in both fluids was significantly elevated.

Biliary bile acid composition was also different during cholestanol administration. A portion of the cholestanol was converted to allocholic acid (16\%) and allochenodeoxycholic acid $(5 \%)$, as illustrated in Fig. 2, which indicates that choles-

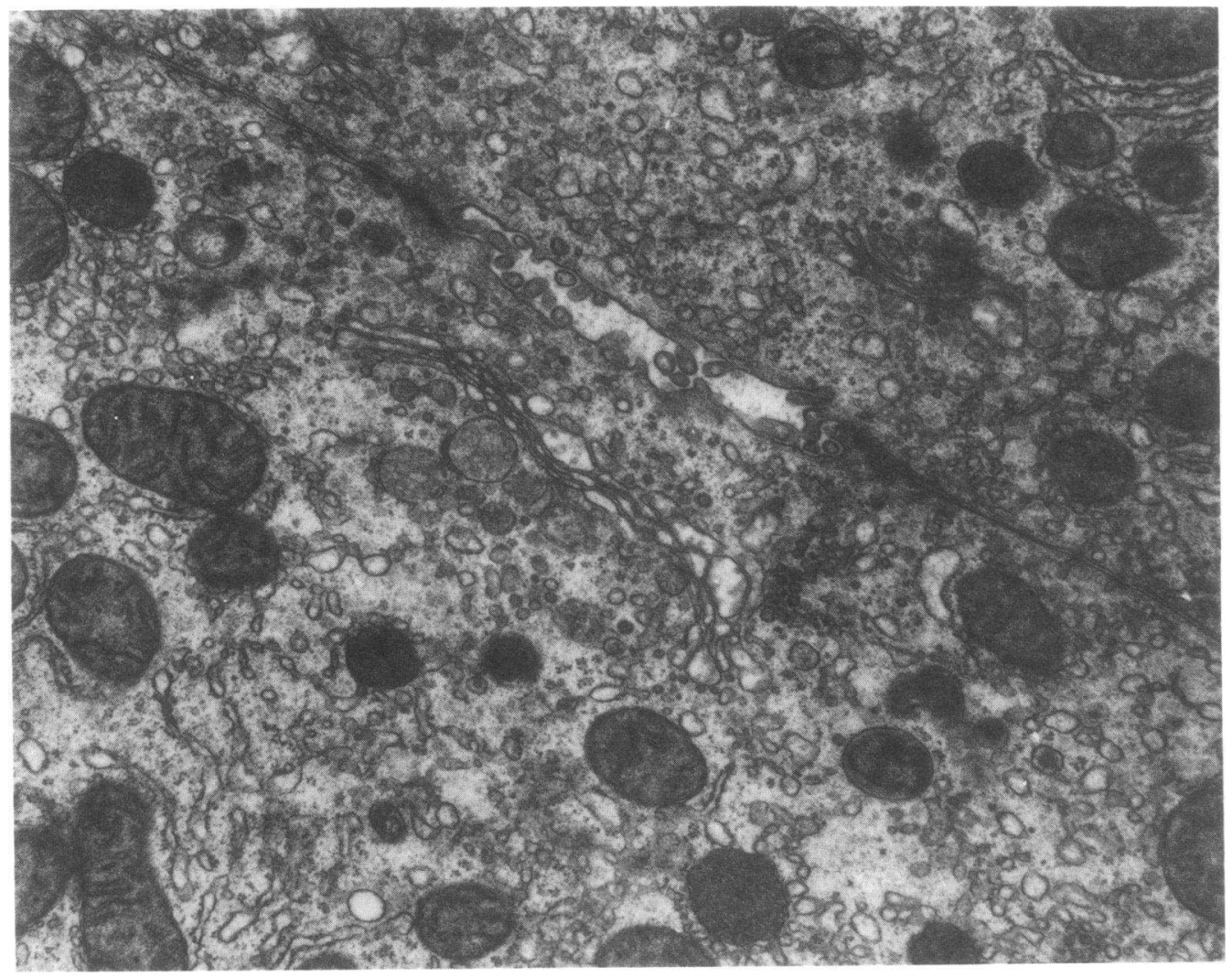

Figure 3. Portion of two hepatocytes in the periportal area of a rat fed cholestanol showing normal Golgi vesicles near bile canaliculus, intact mitochondria, and mild increase in the endoplasmic reticulum. Original magnifcation $\times 4,000$. 

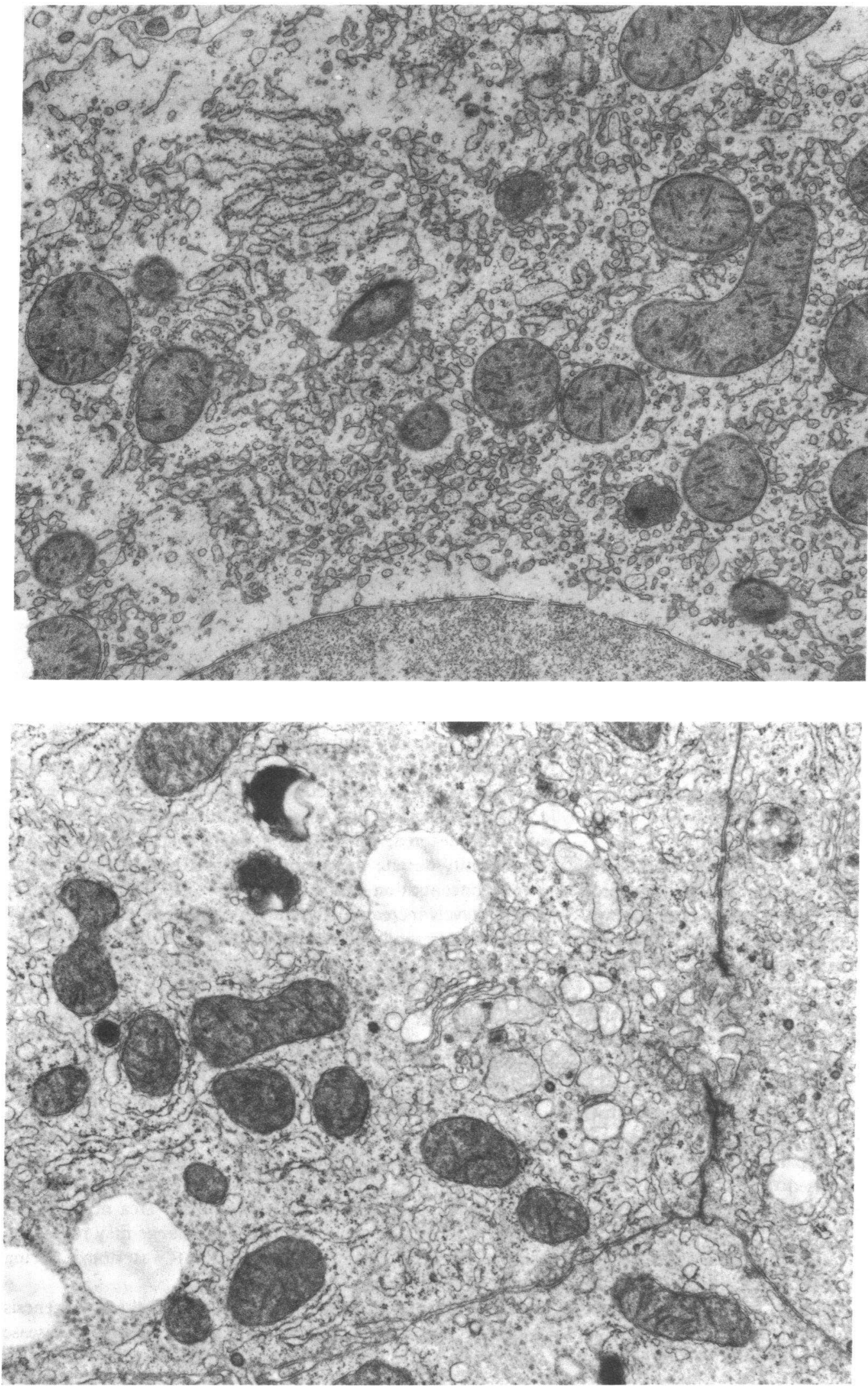

Figure 4. Same rat of Fig. 3. Marked increase in the smooth endoplasmic reticulum of a hepatocyte in the centrilobular area. Original magnification $\times 4,000$.

Figure 5. Rat fed cholesterol. Portion of three hepatocytes from the periportal area showing normal endoplasmic reticulum, mitochondria, and bile canaliculi. The Golgi vesicles appear distended and fed fat droplets are noted. Original magnification $\times 4,000$ 


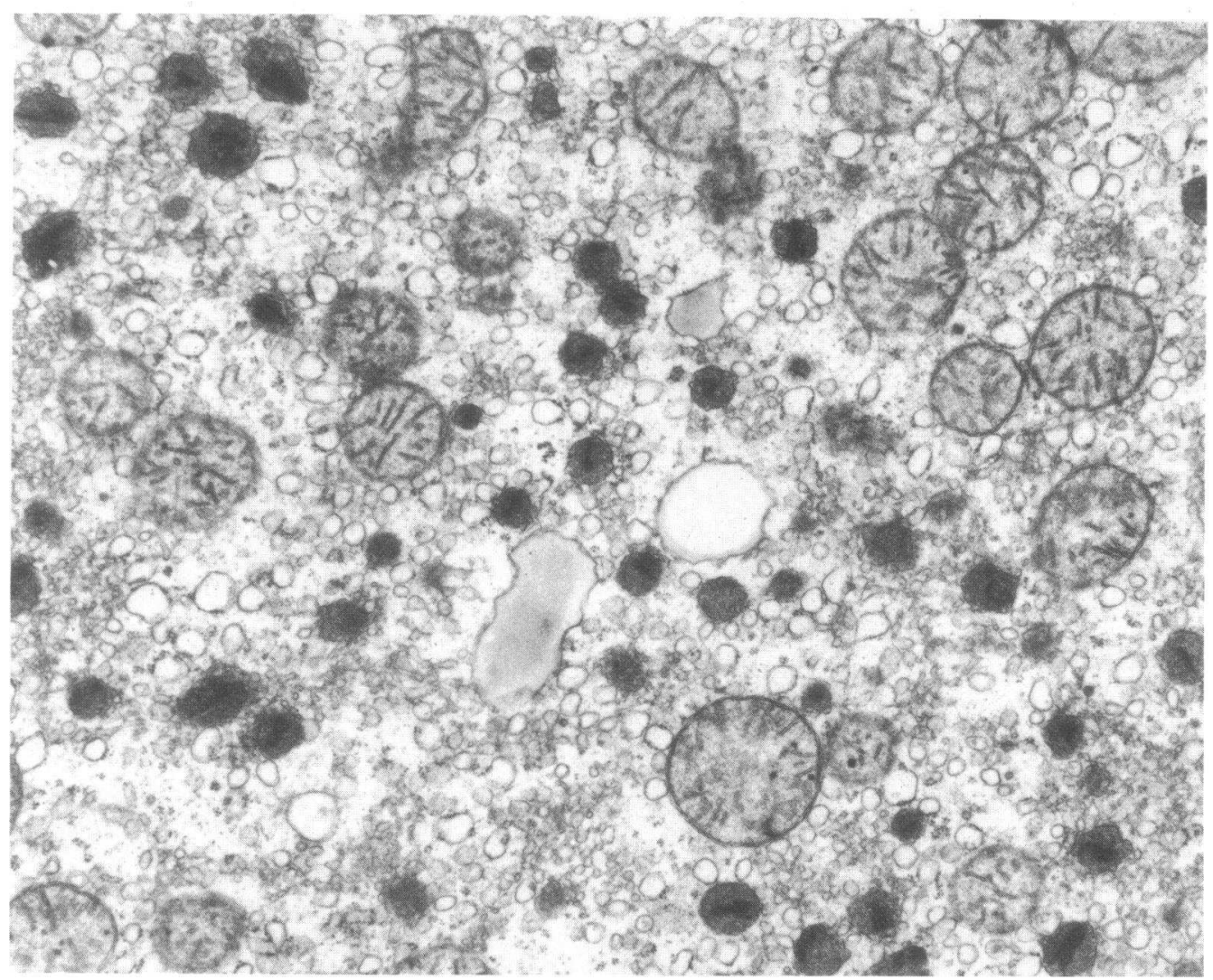

Figure 6. Same rat of Fig. 5. Moderate increase of vesiculated smooth endoplasmic reticulum and significant increase in the peroxisome of a hepatocyte in the centrilobular area. Original magnification $\times 4,000$. tanol, the $5 \alpha$-saturated isomer of cholesterol, could be $7 \alpha$-hydroxylated (27) and transformed by the hepatic enzymes that normally convert cholesterol to bile acids. Alternatively, it is conceivable that separate pathways were involved in the transformation of cholestanol to allo-bile acids (5).

Cholesterol $7 \alpha$-hydroxylase, which is the rate determining enzyme for bile acid synthesis, was not affected by cholesterol or cholestanol feeding (Table V). Although it has been shown that total bile acid synthesis as measured by the sterol balance method increases during cholesterol feeding (8), and indeed similar results were observed in the present study (Table VI), this increase does not result from de novo enzyme synthesis. However, the increase in bile acid synthesis associated with cholesterol feeding may rather reflect greater availability of the substrate for cholesterol $7 \alpha$-hydroxylase (19).

It is important to note that the assay used for measuring cholesterol $7 \alpha$-hydroxylase activity has been improved. A virtually sterol free microsomal preparation (Table V) which contains the active enzyme was used (19). Thus, the results are not confounded by the dilution of the ratioactive exogenous substrate.

The most remarkable observation was the nearly threefold rise in HMG-CoA reductase activity when the diet was supplemented by cholestanol. According to current concepts, cholesterol transported in chylomicron remnants is taken up by the liver and exerts feedback inhibition on HMG-CoA reductase, thereby controlling the endogenous synthesis of cholesterol (28). As expected, when large amounts of cholesterol were fed, HMG-CoA reductase activity declined fivefold (Table IV), supporting this hypothesis. In contrast, when cholestanol was administered, HMG-CoA reductase activity levels rose significantly despite the almost $50 \%$ increase in hepatic total sterol concentration (Table I). As expected, microsomal cholesterol levels increased $40 \%$ during cholesterol feeding, which is probably responsible for the feedback inhibition of HMGCoA reductase activity. When cholestanol was fed, microsomal cholesterol content declined $\sim 8 \%$, which was not statistically significant, without any compensatory increase in cholestanol content. It is possible that the slight increase in microsomal cholestanol concentration could have contributed to the activation of $\mathrm{HMG}-\mathrm{CoA}$ reductase.

Feeding cholestanol or cholesterol to rats, at least for a short period such as $2 \mathrm{wk}$, appears to have no serious impact on the morphologic structure of the hepatic cells. However, as illustrated in Fig. 4, there was a marked increase in the smooth endoplasmic reticulum of the centrilobular area of the hepatocytes of the cholestanol fed rats. This change may reflect the activation of HMG-CoA reductase that was noted during cholestanol feeding.

These results may have some relevance to the pathogenesis of sterol deposits in the rare inherited lipid storage disease cerebrotendinous xanthomatosis. In this disease, large amounts 
of cholestanol are deposited in various tissues associated with enhanced cholesterol synthesis (3). It is conceivable that the accumulation of cholestanol in the liver and tissues of patients with this disease may lead to activation of cholesterol synthesis.

In summary, the present study shows that: $(a)$ cholestanol is absorbed and deposited in the liver; $(b)$ cholestanol competes and reduces cholesterol absorption; and (c) cholestanol feeding is associated with a marked increase in the smooth endoplasmic reticulum of the centrilobular area and leads to the derepression of cholesterol biosynthesis. Thus, despite the structural similarity of cholestanol and cholesterol, considerable biochemical differences exist.

\section{Acknowledgments}

This work was supported by Public Health Service Grants AM-26756, AM-18707, and HL-17818.

\section{References}

1. Bladon, P. 1958. Chemistry. In Cholesterol. R. P. Cook, editor. Academic Press, Inc., New York. 15-115.

2. Werbin, H., I. L. Chaikoff, and M. R. Imada. 1962. 5 $\alpha$-cholestan$3 \beta$-ol: its distribution in tissues and its synthesis from cholesterol in the guinea pig. J. Biol. Chem. 237:2072-2077.

3. Salen, G., S. Shefer, and V. M. Berginer. 1983. Familial diseases with storage of sterols other than cholesterol: cerebrotendinous xanthomathosis and sitosterolemia with xanthomatosis. In The Metabolic Basis of Inherited Disease. J. B. Stanbury, J. B. Wyngaarden, D. S. Fredrickson, J. L. Goldstein, and M. S. Brown, editors. McGraw-Hill, Inc., New York. 713-730.

4. Björkhem, I., and J. Gustafsson. 1971. On the conversion of cholestanol into allo-cholic acid in rat liver. Eur. J. Biochem. 18:207213.

5. Elliott, W. H. 1976. The allo bile acids. In The Hepatobiliary System. W. Taylor, editor. Plenum Press, New York. 469-483.

6. Shefer, S., F. G. Zaki, and G. Salen. 1983. Early morphologic and enzymatic changes in livers of rats treated with chenodeoxycholic and ursodeoxycholic acids. Hepatology (Baltimore). 3:201-208.

7. Nicolau, G., S. Shefer, G. Salen, and E. H. Mosbach. 1974. Determination of hepatic cholesterol $7 \alpha$-hydroxylase activity in man. J. Lipid Res. 15:146-151.

8. Shefer, S., S. Milch, and E. H. Mosbach. 1964. Biosynthesis of $5 \alpha$-cholestan-3 $\beta$-ol in the rabbit and guinea pig. J. Biol. Chem. 239:1731-1736.

9. de Souza, N. J., and W. R. Nes. 1969. Improved separation of sterols by reversed-phase thin-layer chromatography. J. Lipid Res. 10:240-243.

10. Nicolau, G., S. Shefer, G. Salen, and E. H. Mosbach. 1974. Determination of hepatic 3-hydroxy-3-methylglutaryl-CoA reductase activity in man. J. Lipid Res. 15:94-98.

11. Salen, G., and S. M. Grundy. 1973. The metabolism of cholestanol, cholesterol and bile acids in cerebrotendinous xanthomatosis. J. Clin. Invest. 52:2822-2835.

12. Hsia, S. L., J. T. Matschiner, T. A. Mahowald, W. H., Elliott, E. A. Doisy, Jr., S. A. Thayer, and E. A. Doisy. 1957. Bile acids. V. Chemical studies on the new bile acids from the rat and the hog. $J$. Biol. Chem. 225:811-823.

13. Hsia, S. L., J. T. Matschiner, T. A. Mahowald, W. H. Elliott, E. A. Doisy, Jr., S. A. Thayer, and E. A. Doisy. 1957. Bile acids. VI. The structure and synthesis of acid. II. J. Biol. Chem. 226:667-671.

14. Tint, G. S., B. Dayal, A. K. Batta, S. Shefer, F. W. Cheng, G. Salen, and E. H. Mosbach. 1978. Gas-liquid chromatography mass spectrometry in bile alcohols. J. Lipid Res. 19:956-966.

15. Shefer, S., G. Salen, S. Hauser, B. Dayal, and A. K. Batta. 1982. Metabolism of iso-bile acids in the rat. J. Biol. Chem. 257:14011406.

16. Shefer, S., S. Hauser, V. Lapar, and E. H. Mosbach. 1973. Regulatory effects of sterols and bile acids on hepatic 3-hydroxy-3methylglutaryl CoA reductase and cholesterol $7 \alpha$-hydroxylase in the rat. J. Lipid Res. 14:573-580.

17. Grundy, S. M., E. H. Ahrens, and T. A. Miettinen. 1965. Quantitative isolation and gas-liquid chromatographic analysis of total fecal bile acids. J. Lipid Res. 6:397-410.

18. Parmentier, G. G., G. A. Janssen, E. A. Eggermont, and H. J. Eyssen. 1979. $C_{27}$ bile acids in infants with coprostanic acidemia and occurrence of a $3 \alpha, 7 \alpha, 12 \alpha$-trihydroxy- $5 \beta$ - $\mathrm{C}_{29}$ dicarboxylic bile acid as a major component in their serum. Eur. J. Biochem. 102:173-183.

19. Shefer, S., F. W. Cheng, S. Hauser, A. K. Batta, and G. Salen. 1981. Regulation of bile acid synthesis. Measurement of cholesterol $7 \alpha$-hydroxylase activity in rat liver microsomal preparations in the absence of endogenous cholesterol. J. Lipid Res. 22:532-536.

20. Luft, J. 1961. Improvement in epoxy resin embedding methods. J. Biophys. Biochem. Cytol. 9:409-414.

21. Reynolds, E. S. 1963. The use of lead citrate at high $\mathrm{pH}$ as an electron opaque stain in electron microscopy. J. Cell Biol. 17:208212.

22. Borgström, B. 1968. Quantitative aspects of the intestinal absorption and metabolism of cholesterol and $\beta$-sitosterol in the rat. J. Lipid Res. 9:473-481.

23. Shapiro, D. J., and V. W. Rodwell. 1971. Regulation of hepatic 3-hydroxy-3-methylglutaryl coenzyme A reductase and cholesterol synthesis. J. Biol. Chem. 246:3210-3216.

24. Danielsson, H. 1972. Relationship between diurnal variations in biosynthesis of cholesterol and bile acids. Steroids. 20:63-72.

25. Rodwell, V. W., J. L. Nordström, and J. J. Mitschelen. 1976. Regulation of HMG-CoA reductase. Adv. Lipid Res. 14:57-62.

26. Ziller, S. A., Jr., E. A. Doisy, and W. H. Elliott. 1968. Bile acids. XXV. Allochenodeoxycholic acid, a metabolite of $5 \alpha$-cholestan$3 \beta$-ol in the hyperthyroid rat. J. Biol. Chem. 243:5280-5288.

27. Shefer, S., S. Hauser, and E. H. Mosbach. 1968. $7 \alpha$-Hydroxylation of cholestanol by rat liver microsomes. J. Lipid Res. 9:328333.

28. Nervi, F. O., and J. M. Dietschy. 1978. The mechanism of and the interrelationship between bile acid and chylomicron-mediated regulation of hepatic cholesterol synthesis in the liver of the rat. $J$. Clin. Invest. 61:895-908. 